\title{
Anomaly-free dark matter models
}

\author{
Pavel Fileviez Pérez, ${ }^{1, *}$ Elliot Golias, ${ }^{1, \dagger}$ Rui-Hao Li, ${ }^{1, \star}$ Clara Murgui, ${ }^{2, \S}$ and Alexis D. Plascencia ${ }^{1, \|}$ \\ ${ }^{1}$ Physics Department and Center for Education and Research in Cosmology and Astrophysics (CERCA), \\ Case Western Reserve University, Rockefeller Building, 2076 Adelbert Road, Cleveland, Ohio 44106, USA \\ ${ }^{2}$ Departamento de Física Teórica, IFIC, Universitat de Valencia-CSIC, E-46071, Valencia, Spain
}

(Received 12 April 2019; published 15 July 2019)

\begin{abstract}
We investigate the predictions of anomaly-free dark matter models for direct and indirect detection experiments. We focus on gauge theories where the existence of a fermionic dark matter candidate is predicted by anomaly cancellation, its mass is defined by the new symmetry breaking scale, and its stability is guaranteed by a remnant symmetry after the breaking of the gauge symmetry. We find an upper bound on the symmetry breaking scale by applying the relic density and perturbative constraints. The anomaly-free property of the theories allows us to perform a full study of the gamma lines from dark matter annihilation. We investigate the correlation between predictions for final-state radiation processes and gamma lines. Furthermore, we demonstrate that the latter can be distinguished from the continuum gamma-ray spectrum.
\end{abstract}

DOI: $10.1103 /$ PhysRevD.100.015017

\section{INTRODUCTION}

The nature of dark matter $(\mathrm{DM})$ remains one of the most pressing issues in cosmology. In the past few decades, there has been a strong experimental effort to search for the particle nature of dark matter with no positive results so far. The lack of a discovery has pushed the scientific community to perform more general studies on dark matter models. The use of an effective field theory, in which all heavy degrees of freedom except for the dark matter particle are integrated out, covers a large set of dark matter models [1-5]. Nonetheless, it has been shown that the effective field theory approach cannot lead to general conclusions, especially for collider studies where the energy scale can be larger than the cutoff scale.

An alternative framework for the study of different dark matter candidates consists of simplified models [6-12], in which the mediator between the dark matter and the Standard Model (SM) sector is also included in the particle spectrum. In this context, studies of dark matter can be done with only a few parameters, and a systematic study can be performed on a large class of dark matter models. However, these simplified models do not come free of problems.

\footnotetext{
pxf112@case.edu

†ebg23@case.edu

Fx1527@case.edu

\$clara.murgui15@gmail.com

"1exis.plascencia@case.edu
}

Published by the American Physical Society under the terms of the Creative Commons Attribution 4.0 International license. Further distribution of this work must maintain attribution to the author(s) and the published article's title, journal citation, and DOI. Funded by SCOAP ${ }^{3}$.
They present issues of unitarity and gauge anomalies [13-17]. In particular, when considering indirect detection and the predictions for gamma lines from dark matter annihilation, a complete anomaly-free dark matter theory is needed; see e.g., Refs. [18,19] for a detailed discussion.

There exist a large number of theories for dark matter; however, gauge theories can be very appealing due to the fact that the dark matter properties can be defined by a new gauge symmetry in nature. Then, it is possible to have different extensions of the Standard Model where one of the $\mathrm{SM}$ global symmetries is promoted to a local symmetry. For example, if we add three copies of right-handed neutrinos, it is possible to have a theory based on local $B-L$. In this context, it is not possible to predict the existence of a dark matter candidate, but extra fields are required to play this role; see e.g., the study in Ref. [20]. The existence of a fermionic dark matter can be predicted in gauge theories when one of the fields needed for anomaly cancellation is in fact stable and has the right properties to describe the cold dark matter in the Universe. At the same time, the stability of the dark matter candidate can be a consequence of the new gauge symmetry breaking mechanism. The simplest theories we know where one can realize this idea are based on local baryon and lepton numbers [13,21]; for a recent review, see Ref. [22]. The theories based on local baryon number could describe physics at a scale very close to the electroweak scale, and therefore, there is hope to test them in the near future.

In this article, we investigate the dark matter phenomenology in the context of simple theories where baryon number is a local gauge symmetry spontaneously broken at the low scale $[13,21]$. Our dark matter candidate is a Majorana fermion charged under the local $\mathrm{U}(1)_{B}$ gauge 
group, and hence, it has an axial coupling to the new gauge boson $Z_{B}$ present in the theory. This property gives a velocity-suppressed dark matter-nucleon interaction, which means that bounds from direct detection experiments can be avoided. The simplest theories [13,21,23] where baryon number is a local symmetry are very appealing extensions of the Standard Model where the spontaneous breaking of baryon number at a low scale can be understood, the stability of the proton is predicted at any level in perturbation theory, there is a good dark matter candidate in the theory, and there could be interesting mechanisms to explain the baryon asymmetry of the Universe.

We perform a detailed study of the relic density, experimental constraints coming from the LHC, and direct detection experiments. From the requirement of not overproducing dark matter combined with perturbativity, we find an upper bound on the symmetry breaking scale of the theory $\lesssim 28 \mathrm{TeV}$. Therefore, there is a hope to test these theories at the Large Hadron Collider or future colliders. We demonstrate that having a Majorana candidate in this type of theory allows the gamma lines to be visible. This is due to the fact that final-state radiation processes are suppressed. The predictions for gamma lines are very important because gamma lines provide a clean and distinctive signature, and hence, they represent a smoking gun for the discovery of the dark matter.

This article is organized as follows: In Sec. II, we discuss the main features of anomaly-free dark matter models, while in Sec. III, we present a discussion of the simplified model of dark matter; compute the dark matter relic abundance; and study LHC, direct detection, and perturbativity constraints on the model. In Sec. IV, we show that a consistent anomaly-free theory leads to visible gamma lines in the photon spectrum of dark matter annihilation. We present our concluding remarks in Sec. V.

\section{ANOMALY CANCELLATION AND DARK MATTER CANDIDATES}

For simplicity, let us consider a simple Abelian gauge theory and take all the SM fermions to be charged under this new $\mathrm{U}(1)^{\prime}$. Then, the existence of a dark matter candidate can be predicted from the requirement of anomaly cancellation. However, if the new symmetry is anomaly free, e.g., $\mathrm{U}(1)_{L_{i}-L_{j}}$ or $\mathrm{U}(1)_{B-L}$ (with extra righthanded neutrinos), the existence of dark matter cannot be predicted, because there is no need to add new degrees of freedom for anomaly cancellation. In general, we can consider

$$
\begin{array}{rlrl}
Q_{L}^{i} & \sim\left(3,2,1 / 6, n_{Q_{i}}\right), & & u_{R}^{i} \sim\left(3,1,2 / 3, n_{u_{i}}\right), \\
d_{R}^{i} & \sim\left(3,1,-1 / 3, n_{d_{i}}\right), & & \ell_{L}^{i} \sim\left(1,2,-1 / 2, n_{\ell_{i}}\right), \quad \text { and } \\
e_{R}^{i} & \sim\left(1,1,-1, n_{e_{i}}\right),
\end{array}
$$

where each entry in the parenthesis corresponds to the quantum number for each multiplet under the gauge groups
$\left[\mathrm{SU}(3)_{C}, \mathrm{SU}(2)_{L}, \mathrm{U}(1)_{Y}, \mathrm{U}(1)_{B-L}\right]$ and $i=1,2,3$ is the family index. Finding a simple solution to the anomaly cancellation conditions in agreement with all experimental bounds is a nontrivial exercise. After the discovery of the SM Higgs boson, it is possible to include extra fermions in a theory, but they should be vectorlike under the SM gauge group, and if they are chiral under the new gauge symmetry, they must acquire mass from the symmetry breaking mechanism. It is possible to find different solutions for anomaly cancellation, but generically, these theories will have the following general features:

(1) There is an extra electrically neutral field, $\chi_{L(\text { or } R)} \sim$ $\left(1,1,0, n_{\chi}\right)$, which plays a role in the cancellation of anomalies. This field can be stable, and hence a good cold dark matter candidate.

(2) The mass of the DM candidate is determined by the new symmetry breaking scale.

(3) The stability of the DM candidate is a natural consequence of the symmetry breaking. In the scenario where the DM is a Majorana fermion, the Abelian symmetry is broken to a $Z_{2}$ discrete symmetry, while in the Dirac case, the stability is determined by an anomaly-free global symmetry.

(4) The masses of the fermions required for anomaly cancellation have an upper bound defined by the new symmetry scale.

(5) An upper bound on the symmetry breaking scale can be found by applying cosmological relic density constraints.

Perhaps the simplest cases correspond to the theories where the baryon and/or lepton numbers are local symmetries [13,21]. In this article, for simplicity, we focus on the case where the new symmetry is the local baryon number, $\mathrm{U}(1)_{B}$, because this theory can describe new physics at a scale very close to the electroweak scale in agreement with all experimental constraints. In our study, we will investigate the properties of the Majorana dark matter candidates, since they are predicted in both theories proposed in Ref. [13,21].

\section{MAJORANA LEPTOPHOBIC DARK MATTER}

In this article, we investigate the properties of a Majorana WIMP dark matter candidate in theories where baryon number is a local symmetry spontaneously broken at the low scale. Here we discuss the main properties of our dark matter candidate. The relevant Lagrangian for our discussion is given by

$$
\begin{aligned}
\mathcal{L} \supset & -g_{B} n_{\chi} \bar{\chi} \gamma^{\mu} \gamma^{5} \chi Z_{\mu}^{B}-g_{B} \bar{f}\left(n_{V}^{f} \gamma^{\mu}+n_{A}^{f} \gamma^{\mu} \gamma^{5}\right) f Z_{\mu}^{B} \\
& -\lambda_{i} \bar{\chi} \chi h_{i}-\frac{1}{2} M_{\chi} \chi^{T} C \chi,
\end{aligned}
$$

where $\chi=\chi^{C}$ is a Majorana fermion. The first term defines the interaction between dark matter $\chi$ and the new gauge 
boson $Z_{B}$, which mediates the new baryonic force. Here $g_{B}$ is the new gauge coupling, and the fermions $f$ can be any SM quark or the new fermions needed for anomaly cancellation. The coefficients $\lambda_{i}$ define the interaction between the Higgses present in the theory and the DM candidate. Typically, one needs only two Higgses in these theories: the SM Higgs and the new Higgs needed to generate mass for the new gauge boson and fermions needed for anomaly cancellation. Therefore, the mechanism of spontaneous symmetry breaking needs to be applied in these theories; see Refs. [13,21] for details.

In order to study the dark matter phenomenology, the most relevant part of the Lagrangian in the theories proposed in Refs. [13,21] is given by

$$
\begin{aligned}
\mathcal{L} \supset & \frac{3}{4} g_{B} \bar{\chi} \gamma^{\mu} \gamma^{5} \chi Z_{\mu}^{B}-\frac{1}{3} g_{B} \bar{q} \gamma^{\mu} q Z_{\mu}^{B}+\frac{M_{\chi}}{2 v_{B}} \sin \theta_{B} \bar{\chi} \chi h_{1} \\
& -\frac{M_{\chi}}{2 v_{B}} \cos \theta_{B} \bar{\chi} \chi h_{2}-\frac{1}{2} M_{\chi} \bar{\chi} \chi,
\end{aligned}
$$

where we have assumed the simple case where the DM is the Majorana fermion $\chi=\chi_{L}+\left(\chi_{L}\right)^{C}$ and neglect the mixing with the other fermions present in the theory for simplicity. The factor $3 / 4$ is the baryon number of the dark matter divided by 2 , where the latter comes from the $1 / 2$ factor in the projector. The gauge boson has vector coupling to quarks, since $Z_{B}$ is associated with baryon number. In this paper, we will focus on this scenario, since it is realized in both models in Refs. [13,21]. In the above equation, $\theta_{B}$ is the mixing angle between the SM Higgs and the new Higgs present in the theory to break the local baryon number symmetry, $S_{B} \sim(1,1,0,3)$. In the above equation, $h_{1}$ and $h_{2}$ are the physical states present in the theory.

The new symmetry breaking scale $v_{B}$ can be replaced by $v_{B}=M_{Z_{B}} / 3 g_{B}$, where $M_{Z_{B}}$ is the mass of the new gauge boson. Henceforth, we set the SM Higgs boson mass to $M_{h_{1}}=125.09 \mathrm{GeV}$ and $v_{H}=246.22 \mathrm{GeV}$. Then, this simplified model contains five free parameters:

$$
M_{\chi}, \quad M_{Z_{B}}, \quad M_{h_{2}}, \quad \theta_{B}, \quad g_{B} .
$$

In Ref. [24], we have discussed in detail the experimental bounds on $M_{Z_{B}}$ and $\theta_{B}$. In the next section, we will discuss the impact of the cosmological bounds and the possibility to find an upper bound on the symmetry breaking scale. The simplified model described in Eq. (2) can arise from the following gauge-invariant Lagrangian:

$$
\mathcal{L} \supset i \bar{\chi}_{L} \gamma^{\mu} D_{\mu} \chi_{L}-\left(\frac{\lambda_{\chi}}{\sqrt{2}} \chi_{L}^{T} C \chi_{L} S_{B}^{*}+\text { H.c. }\right)
$$

where $D^{\mu} \chi_{L}=\partial^{\mu} \chi_{L}+i\left(3 g_{B} / 2\right) Z_{B}^{\mu} \chi_{L}$. Defining a Majorana field $\chi=\chi_{L}+\left(\chi_{L}\right)^{C}$, one can obtain the terms in Eq. (2), whereas the dark matter mass is given by

$$
M_{\chi}=\lambda_{\chi} v_{B} .
$$

The scalar potential is given by

$$
\begin{aligned}
V\left(H, S_{B}\right)= & -\mu_{H}^{2} H^{\dagger} H-\mu_{B}^{2} S_{B}^{\dagger} S_{B}+\lambda_{H}\left(H^{\dagger} H\right)^{2} \\
& +\lambda_{B}\left(S_{B}^{\dagger} S_{B}\right)^{2}+\lambda_{H B}\left(H^{\dagger} H\right)\left(S_{B}^{\dagger} S_{B}\right),
\end{aligned}
$$

where $H$ corresponds to the SM Higgs doublet and $S_{B}$ is charged under the $\mathrm{U}(1)_{B}$ group. In the zero-temperature vacuum of the theory, both fields acquire a nonzero vacuum expectation value, and we can write

$$
S_{B}=\frac{1}{\sqrt{2}}\left(s_{B}+v_{B}\right), \quad H=\frac{1}{\sqrt{2}}\left(\begin{array}{c}
0 \\
h+v_{H}
\end{array}\right),
$$

where the Higgs doublet has been written in the unitary gauge. This leads to mixing among both scalars, and hence, the mass matrix needs to be diagonalized in order to find the physical states. The latter are given by

$$
\begin{aligned}
& h_{1}=h \cos \theta_{B}-s_{B} \sin \theta_{B}, \\
& h_{2}=s_{B} \cos \theta_{B}+h \sin \theta_{B},
\end{aligned}
$$

where the scalar mixing angle can be written in terms of the scalar quartic couplings and the vacuum expectation values:

$$
\tan 2 \theta_{B}=\frac{\lambda_{H B} v_{H} v_{B}}{\lambda_{B} v_{B}^{2}-\lambda_{H} v_{H}^{2}} .
$$

In order to perform this study, we need to understand all perturbative bounds on the free parameters of the model. In Ref. [24], we have pointed out the need to impose the perturbative bounds on the scalar couplings in the Higgs sector,

$$
\begin{aligned}
& \lambda_{H}=\frac{1}{2 v_{H}^{2}}\left(M_{h_{1}}^{2} \cos ^{2} \theta_{B}+M_{h_{2}}^{2} \sin ^{2} \theta_{B}\right) \leq 4 \pi, \\
& \lambda_{B}=\frac{1}{2 v_{B}^{2}}\left(M_{h_{1}}^{2} \sin ^{2} \theta_{B}+M_{h_{2}}^{2} \cos ^{2} \theta_{B}\right) \leq 4 \pi, \\
& \lambda_{H B}=\frac{1}{v_{H} v_{B}}\left(M_{h_{2}}^{2}-M_{h_{1}}^{2}\right) \sin \theta_{B} \cos \theta_{B} \leq 4 \pi .
\end{aligned}
$$

To ensure vacuum stability of the scalar potential, we impose

$$
\lambda_{H}, \lambda_{B}>0 \text { and } \lambda_{H B}>-2 \sqrt{\lambda_{H} \lambda_{B}} \text {; }
$$

for more details, see Ref. [24]. For consistency of our calculation, the gauge coupling $g_{B}$ must remain perturbative. If we revisit all the gauge interactions present in the theory, symbolically we have that $\bar{q} Z_{B} q, \bar{\chi} Z_{B} \chi$, and $Z_{B} Z_{B} S_{B} S_{B}$, and we find that the strongest upper bound 

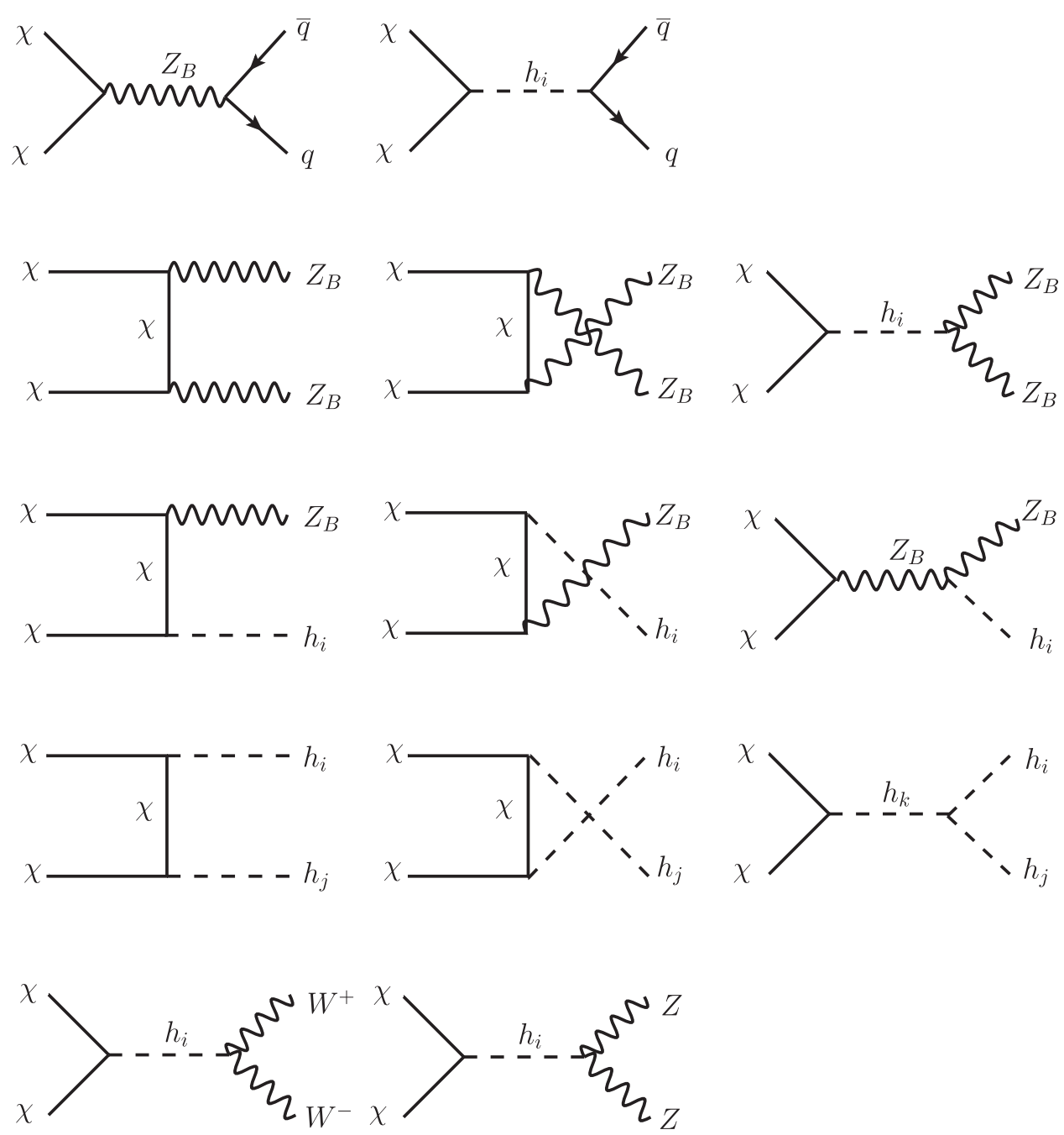

FIG. 1. Feynman diagrams for the dark matter annihilation channels.

on $g_{B}$ is coming from the $Z_{B} Z_{B} S_{B} S_{B}$ interaction, and it reads as $g_{B} \leq \sqrt{2 \pi} / 3$. Following the notation above, the perturbative bound on the Yukawa coupling between the dark matter and the Higgses is $\lambda_{x}<2 \sqrt{\pi}$.

\section{A. Relic density}

The knowledge of all the details of the model allows us to investigate in detail the dark matter annihilation channels. In this context, the leptophobic dark matter can have the following annihilation channels:

$$
\chi \chi \rightarrow \bar{q} q, Z_{B} Z_{B}, Z_{B} h_{1}, Z_{B} h_{2}, h_{1} h_{1}, h_{1} h_{2}, h_{2} h_{2}, W W, Z Z .
$$

The channels $Z_{B} h_{1}, h_{1} h_{1}, h_{1} h_{2}, W W$, and $Z Z$ are suppressed by the mixing angle $\theta_{B}$. In Fig. 1, we show the Feynman graphs for each channel. For our numerical study, we implement the model in LANHEP 3.2 [25] and perform the calculation of $\Omega_{\mathrm{DM}} h^{2}$ using MicROMEGAs 5.0.6 [26].
Moreover, we perform an independent calculation in Mathematica.

In Fig. 2, we present results for the relic density in the $M_{\chi}$ versus $M_{Z_{B}}$ plane. The dark blue line corresponds to the measured relic abundance by the Planck satellite measurement of $\Omega_{\mathrm{DM}} h^{2}=0.1197 \pm 0.0022$ [27]. The region shaded in light blue overproduces the dark matter relic density, and it is ruled out unless the thermal history of the Universe is modified. We show results for four different scenarios: $g_{B}=0.1$ (top left), $g_{B}=0.3$ (top right), $g_{B}=$ 0.5 (bottom left), and $g_{B}=\sqrt{2 \pi} / 3$ (bottom right).

The $Z_{B}$ mediator has direct coupling to quarks and would appear as a resonance in dijet searches at the LHC. In our work, we apply the bounds from CMS and ATLAS [24] and present the excluded regions in purple bands in Figs. 2 and 3. These bounds have a strong dependence on the coupling $g_{B}$ and disappear for $g_{B} \leq 0.1$. Additionally, the leptophobic gauge boson will develop a kinetic mixing with hypercharge (and hence the SM $Z$ boson) from radiative corrections. This, in turn, will modify electroweak 

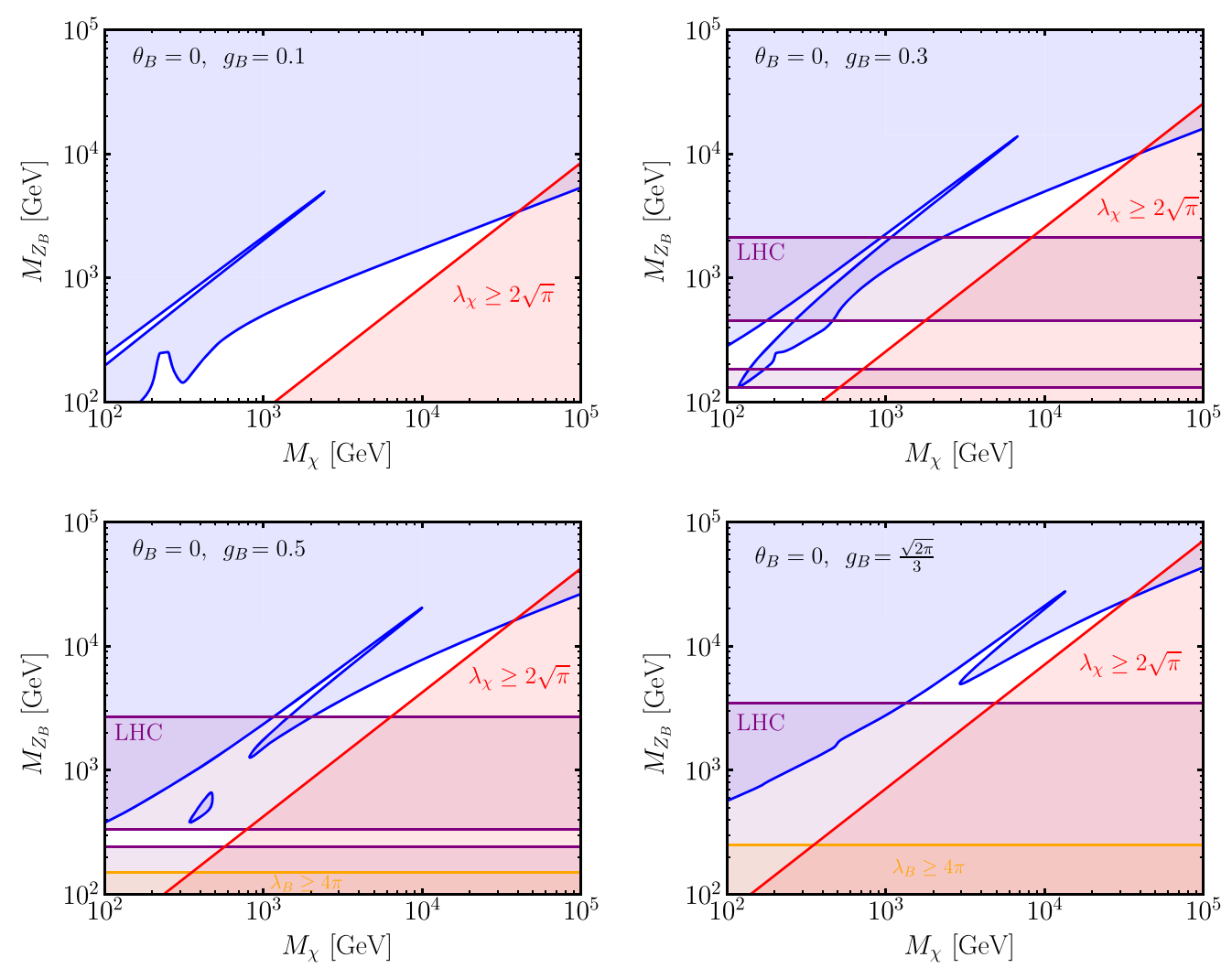

FIG. 2. Parameter space allowed by the relic density constraint, LHC bounds, and perturbative bounds for four different scenarios: $g_{B}=0.1$ (top left), $g_{B}=0.3$ (top right), $g_{B}=0.5$ (bottom left), and $g_{B}=\sqrt{2 \pi} / 3$ (bottom right). We take $M_{h_{2}}=500 \mathrm{GeV}$ and no mixing angle. The region shaded in blue overproduces dark matter $\Omega_{\mathrm{DM}} h^{2}>0.12$, and the region in red (yellow) is excluded by the perturbative bound on the Yukawa coupling $\lambda_{\chi}$ (scalar coupling $\lambda_{B}$ ). The horizontal purple bands are excluded by the LHC bounds on the leptophobic gauge boson mass.

precision observables which have been measured to great accuracy. However, as has been shown in Ref. [28], these bounds become relevant only for $M_{Z_{B}} \lesssim 100 \mathrm{GeV}$; therefore, we do not consider them for our analysis. The mass of the second Higgs is fixed to $M_{h_{2}}=500 \mathrm{GeV}$ and the mixing angle to $\theta_{B}=0$. The region shaded in red is ruled out by the perturbative bound on the Yukawa coupling, $\lambda_{\chi}<2 \sqrt{\pi}$. Similarly, the yellow region is ruled out by the perturbative bound on the scalar quartic coupling, $\lambda_{B}<4 \pi$.

The choice of the perturbative limit $g_{B}=\sqrt{2 \pi} / 3$ is responsible for the upper bound on the symmetry breaking scale, $M_{Z_{B}} \leq 28 \mathrm{TeV}$. This striking feature tells us that one can hope to test or rule out this theory in the near future. The choice of zero scalar mixing can be motivated as follows: In the SM, the electroweak phase transition occurs around $T_{\mathrm{EW}} \approx 160 \mathrm{GeV}$. For masses above the TeV scale (which is the region preferred after considering LHC bounds), we find the dark matter freeze-out temperature to be $x_{f} \approx 26-29$. Consequently, for $M_{\chi} \gtrsim 5 \mathrm{TeV}$, the freeze-out temperature is above the electroweak phase transition, and hence, at the time when DM freezes out, the Higgs field has a vacuum expectation value of zero and there is no scalar mixing. It is important to mention that the bounds from the relic density constraint are very similar in the case where one considers a nonzero mixing angle.

In order to develop a better understanding of our results, we present in Fig. 3 the parameter space allowed by the relic density constraint. We color each region depending on which annihilation is the dominant one-namely, the one that gives the largest contribution to the relic abundance at freeze-out. The region in dark blue corresponds to the region in parameter space where annihilation into quarks is the dominant channel; this happens near the resonance $M_{\chi} \approx M_{Z_{B}} / 2$. The region in light blue is where the annihilation into $Z_{B} Z_{B}$ is the most dominant one. This occurs due to the resonance $\chi \chi \rightarrow h_{2}^{*} \rightarrow Z_{B} Z_{B}$ when $M_{\chi} \approx M_{h_{2}} / 2 \approx$ $250 \mathrm{GeV}$. This resonant behavior can be easily appreciated in the upper-left panels in Figs. 2 and 3. The resonance is cut close to the diagonal $M_{\chi} \approx M_{Z_{B}}$, because the $Z_{B} Z_{B}$ channel becomes kinematically closed above.

The region in which the $h_{2} h_{2}$ annihilation channel is the dominant one is colored in dark green. The latter is velocity suppressed and at freeze-out $v_{\mathrm{DM}} \approx 0.3$; consequently, this channel is dominant only in a small region in the parameter space. Once the $Z_{B} h_{2}$ channel becomes kinematically open, it becomes the dominant channel, as illustrated by the 

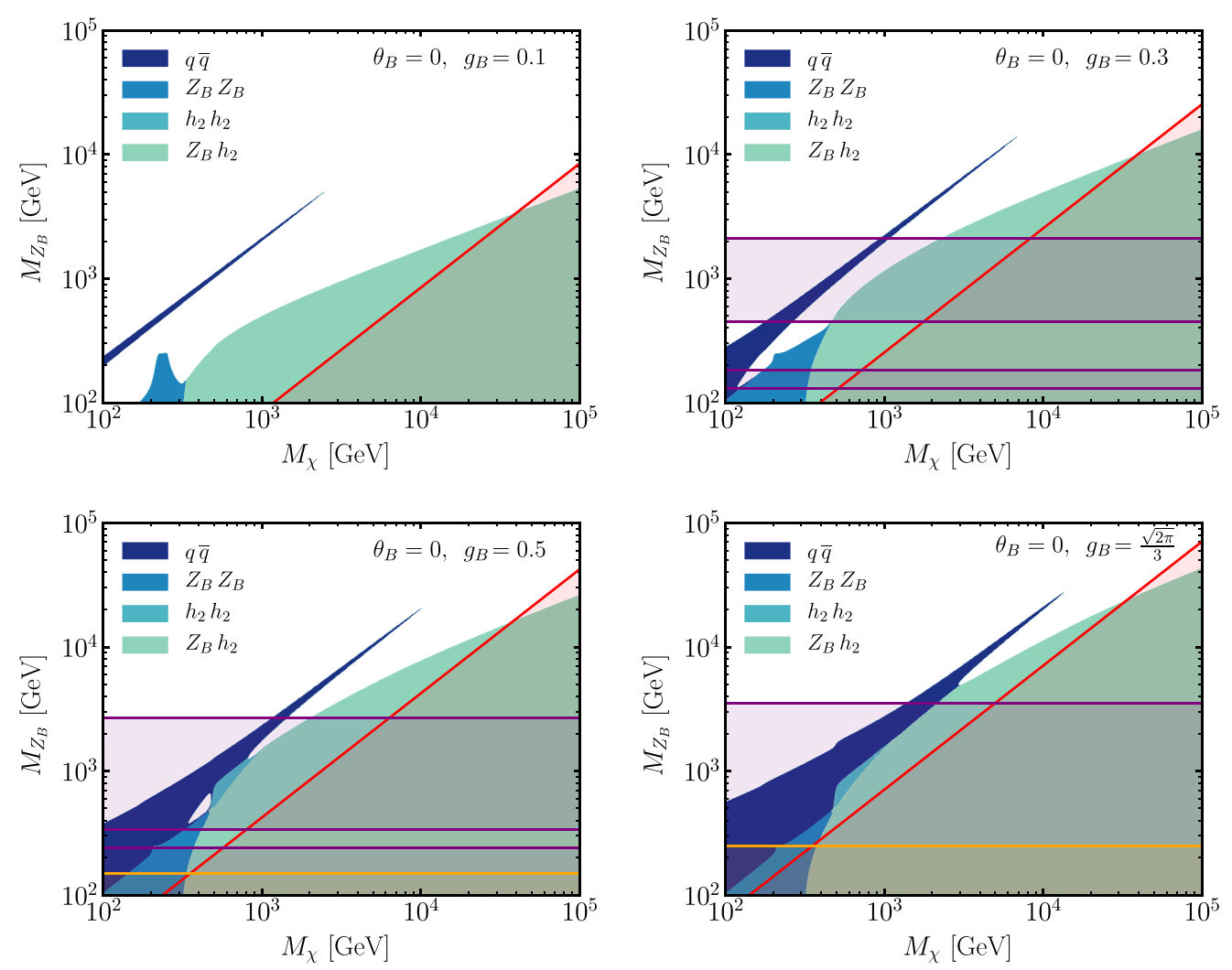

FIG. 3. Regions in the $M_{\chi}$ versus $M_{Z_{B}}$ plane that satisfy the relic density constraint $\Omega_{\mathrm{DM}} h^{2} \leq 0.12$; the colors indicate which annihilation channel is the dominant one. We fix $M_{h_{2}}=500 \mathrm{GeV}$ and $\theta_{B}=0$. The horizontal purple bands are excluded by dijet searches at the LHC. The region in red (yellow) is excluded by the perturbative bound on the Yukawa coupling $\lambda_{\chi}$ (scalar quartic coupling $\lambda_{B}$ ).

region in light green. As can be appreciated, the upper bound on the gauge boson mass, $M_{Z_{B}} \leq 28 \mathrm{TeV}$, is defined by the annihilation into quarks, while the upper bound on the dark matter mass, $M_{\chi} \leq 34 \mathrm{TeV}$, is determined by the $Z_{B} h_{2}$ channel.

\section{B. Direct detection}

Our dark matter candidate can interact with quarks in the nucleon via exchange of a leptophobic gauge boson or one of the physical scalars through the Higgs mixing. These two processes do not interfere with each other, and the spinindependent cross section between $\chi$ and a nucleon can be written as $\sigma_{\chi N}^{\mathrm{TOT}}=\sigma_{\chi N}\left(Z_{B}\right)+\sigma_{\chi N}\left(h_{i}\right)$, where

$$
\sigma_{\chi N}^{\mathrm{SI}}\left(Z_{B}\right)=\frac{27}{8 \pi} \frac{g_{B}^{4} M_{N}^{2}}{M_{Z_{B}}^{4}} v^{2},
$$

$\sigma_{\chi N}^{\mathrm{SI}}\left(h_{i}\right)=\frac{72 G_{F}}{\sqrt{2} 4 \pi} \sin ^{2} \theta_{B} \cos ^{2} \theta_{B} M_{N}^{4} \frac{g_{B}^{2} M_{\chi}^{2}}{M_{Z_{B}}^{2}}\left(\frac{1}{M_{h_{1}}^{2}}-\frac{1}{M_{h_{2}}^{2}}\right)^{2} f_{N}^{2}$,

$M_{N}$ corresponds to the nucleon mass, $G_{F}$ is the Fermi constant, and for the effective Higgs-nucleon-nucleon coupling we take $f_{N}=0.3$ [29]. The axial coupling between $\chi$ and $Z_{B}$ leads to velocity suppression of the cross section, and we can write

$$
\sigma_{\chi N}^{\mathrm{TOT}}=\sigma_{\chi N}\left(h_{i}\right)+\sigma_{\chi N}^{0}\left(Z_{B}\right) v^{2} .
$$

The bounds coming from direct detection experiments are obtained under the assumption that the leading order in the cross section is velocity independent. In order to apply these bounds, we proceed as follows:

$$
\sigma_{\chi N}\left(h_{i}\right)+\sigma_{\chi N}^{0}\left(Z_{B}\right) v_{\text {eff }}^{2} \leq \sigma_{\chi N}^{\text {DDexp }},
$$

where $\sigma_{\chi N}^{\text {DDexp }}$ is the upper bound on the scattering cross section given by the direct detection experiments, and the effective velocity is given by the ratio $\overline{v^{3}} / \bar{v}$, where the average velocity is the velocity of the dark matter convoluted with a Maxwell-Boltzmann distribution. We find that $v_{\text {eff }} \approx 0.001 c$.

Collider searches of a new scalar that mixes with the Higgs combined with measurements of Higgs properties provide constraints on the mixing angle [30,31]. In our study, the mass of the second Higgs is above the electroweak scale, and hence, we take the bound $\sin \theta_{B} \leq 0.3$. In Fig. 4, we present the predictions for the spin-independent 


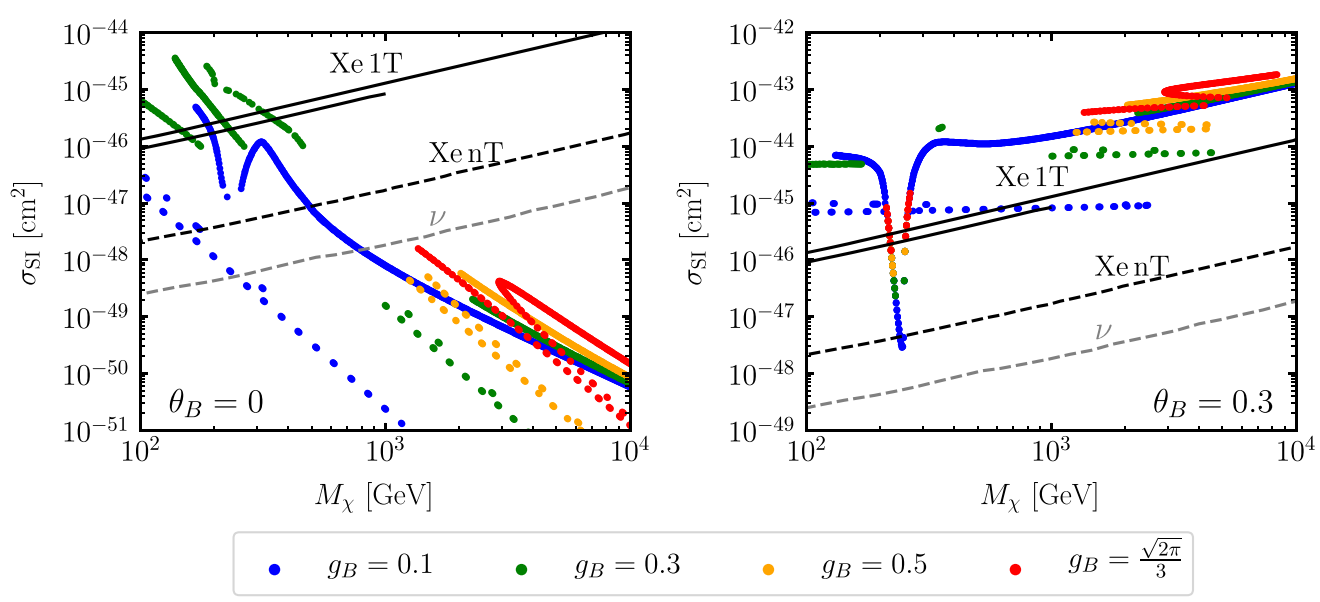

FIG. 4. Predictions for the direct detection spin-independent cross section as a function of the dark matter mass. In the left (right) panel, we present the predictions for $\theta_{B}=0\left(\theta_{B}=0.3\right)$. All points agree with the measured relic abundance by the Planck satellite $\Omega_{\mathrm{DM}} h^{2}=0.1197 \pm 0.0022$ [27] and satisfy constraints from the LHC. The solid black lines show current experimental bounds from Xenon-1T [32,33], the dashed black line shows the projected sensitivity for Xenon-nT [34], and the dashed gray line shows the coherent neutrino scattering limit [35].

cross section for different values of the gauge coupling. In order to select the points, we proceed as follows. First, we select those points that give the measured relic abundance of $\Omega_{\mathrm{DM}} h^{2}=0.1197 \pm 0.0022$. Then, we remove those points that do not satisfy LHC and/or perturbativity bounds. This is the reason behind the discontinuities in the points shown.

The left panel shows the results for $\theta_{B}=0$ and, as expected, due to the velocity suppression, the points with dark matter mass above the $\mathrm{TeV}$ scale lie below the neutrino floor and will escape detection from future experiments. In contrast, for the maximal mixing allowed, $\theta_{B}=0.3$, all the points that saturate the relic density are ruled out by direct detection bounds except for those that lie close to the $h_{2}$ resonance $\left(M_{\chi} \approx M_{h_{2}} / 2\right)$; this is shown in the right panel in Fig. 4. For intermediate values of the mixing angle, the predictions lie in between these two regions, and thus, some of these points will be probed by future direct detection experiments, such as Xenon-nT [34].

\section{GAMMA LINES FROM LEPTOPHOBIC DM}

The model discussed so far suffers from gauge anomalies. In this section, we will argue that the extra fermions required to make the theory anomaly free also lead to dark matter annihilation into photons. Consequently, the photon spectrum will contain features that could be observed in future telescopes. In order to predict the cross sections for

$$
\chi \chi \rightarrow \gamma \gamma, \gamma Z, \gamma h
$$

we need to study anomaly-free gauge theories based on $\mathrm{U}(1)_{B}$. These processes are quantum mechanical and can be predicted only after we understand the anomaly cancellation. In this section, we study the predictions for the gamma lines in the simple models proposed in Refs. [13,21].

Gamma lines are typically suppressed with respect to other processes contributing to the continuum, such as final-state radiation (FSR), and hence, it is hard to observe them. However, it is possible to have scenarios in which the processes that contribute to the continuum close to the gamma lines are highly suppressed and the gamma lines become visible. This can be easily understood in terms of the energy of the gamma lines,

$$
E_{i}^{\gamma}=M_{\mathrm{DM}}\left(1-\frac{M_{i}^{2}}{4 M_{\mathrm{DM}}^{2}}\right),
$$

where $M_{i}=0, M_{Z}, M_{h}$ for the corresponding gamma lines, DM DM $\rightarrow \gamma \gamma, \gamma Z, \gamma h$. At the same time, there are processes such as final-state radiation processes, $\mathrm{DM} \mathrm{DM} \rightarrow \mathrm{SM} \mathrm{SM} \gamma$, which occur at tree level and could spoil the visibility of the gamma lines because the maximal energy of the photon in this case is given by

$$
E_{\max }^{\gamma}=M_{\mathrm{DM}}\left(1-\frac{M_{\mathrm{SM}}^{2}}{M_{\mathrm{DM}}^{2}}\right) .
$$

Thus, when $M_{\mathrm{DM}} \gg M_{\mathrm{SM}}$, the gamma line visibility is spoiled if the FSR processes are not suppressed, because $E_{\max }^{\gamma} \approx E_{i}^{\gamma}$. There are many studies in the literature where the predictions for the gamma spectrum from dark matter annihilation have been investigated; we refer the reader to Ref. [36] for a detailed discussion of different scenarios. Recently, we have investigated dark matter models where the gamma lines are visible due to the fact that the FSR processes are naturally suppressed [19,37]. For studies of models that give rise to gamma-ray lines, see Refs. [38-43]. 
In this article, we investigate the predictions for gamma lines in simple extensions of the Standard Model, our DM candidate is a Majorana fermion, and the FSR processes are velocity suppressed. Consequently, there is hope to observe the gamma lines, which is a striking feature of these models. There is a simple way to understand the velocity suppression of some DM annihilation channels. In our case, the DM annihilation can be mediated only by the $Z^{\prime}$ and the Higgses. For example, for the DMDM $\rightarrow \bar{q} q$ channel, the relevant operator is $\left(\bar{\chi} \gamma^{\mu} \gamma^{5} \chi\right)\left(\bar{q} \gamma_{\mu} q\right)$ and the amplitude is proportional to $\bar{v}_{\chi}\left(p_{2}\right) \gamma^{\mu} \gamma^{5} u_{\chi}\left(p_{1}\right)$. In the nonrelativistic limit, the term $\bar{v}_{\chi}\left(p_{2}\right) \gamma^{i} \gamma^{5} u_{\chi}\left(p_{1}\right)$ is proportional to the velocity $v^{i}$, while $\bar{v}_{\chi}\left(p_{2}\right) \gamma^{0} \gamma^{5} u_{\chi}\left(p_{1}\right)$ is not velocity suppressed. However, since $\bar{u}_{q}\left(p_{4}\right) \gamma_{0} v_{q}\left(p_{3}\right)=0$ when $\vec{p}_{3}=-\vec{p}_{4}$, the full amplitude is velocity suppressed. In general, one can say that the amplitude of any process, $\mathrm{DMDM} \rightarrow\left(Z^{\prime}\right)^{*} \rightarrow$ any, is velocity suppressed if there is no contribution from the term $\bar{v}_{\chi}\left(p_{2}\right) \gamma^{0} \gamma^{5} u_{\chi}\left(p_{1}\right)$.

For the gamma lines, DMDM $\rightarrow\left(Z^{\prime}\right)^{*} \rightarrow \gamma X$, where $X=\gamma, h, Z$, the above argument can be applied. These processes are not velocity suppressed when the amplitude is proportional to $\bar{v}_{\chi}\left(p_{2}\right) \gamma_{0} \gamma^{5} u_{\chi}\left(p_{1}\right) \delta \Gamma_{Z^{\prime} X \gamma}^{0 \sigma \rho}$, where $\delta \Gamma_{Z^{\prime} X \gamma}^{\mu \sigma \rho}$ is the effective $Z^{\prime} X \gamma$ coupling computed in Ref. [18]. Moreover, this effective vertex violates parity, and hence, it can only be obtained from fermions in the loop that have an axial interaction with the new gauge boson, i.e., $\bar{\psi} \gamma^{\mu} \gamma^{5} \psi Z_{\mu}^{\prime}$. This simple argument can be applied to any annihilation channel with any number of fields in the final state. See also Ref. [44] for the study of properties of the amplitude in different DM models.

\section{A. Theories for baryon number}

We have discussed the main features of simplified models with a baryonic Majorana dark matter. This type of dark matter candidate has been predicted in models where the baryon number is defined as a local symmetry. The simplest models based on $\mathrm{U}(1)_{B}$ with a Majorana dark matter have been pointed out in Refs. [13,21]. In Tables I and II, we show the extra fermionic representations in these theories.

Model I: Ref. [13] proposed a simple anomaly-free theory for the spontaneous breaking of local baryon number where the anomalies are canceled with the fields listed in Table I.

The relevant Lagrangian for our discussion is given by

$$
\begin{aligned}
\mathcal{L} \supset & y_{1} \bar{\Psi}_{L} H \eta_{R}+y_{2} \bar{\Psi}_{L} \tilde{H}_{\chi_{R}}+y_{3} \bar{\Psi}_{R} H \eta_{L}+y_{4} \bar{\Psi}_{R} \tilde{H} \chi_{L} \\
& +\lambda_{1} \bar{\Psi}_{L} \Psi_{R} S_{B}^{*}+\lambda_{2} \bar{\eta}_{R} \eta_{L} S_{B}^{*}+\lambda_{3} \bar{\chi}_{R} \chi_{L} S_{B}^{*} \\
& +\frac{\lambda_{\chi}}{\sqrt{2}} \chi_{L} \chi_{L} S_{B}^{*}+\lambda_{5} \chi_{R} \chi_{R} S_{B}+\text { H.c. },
\end{aligned}
$$

where $H \sim(1,2,1 / 2,0)$ and $S_{B} \sim(1,1,0,3)$. In general, the DM candidate can be a linear combination of all neutral
TABLE I. Fermionic representations in the model proposed in Ref. [13].

\begin{tabular}{lcccc}
\hline \hline Fields & $\mathrm{SU}(3)_{C}$ & $\mathrm{SU}(2)_{L}$ & $\mathrm{U}(1)_{Y}$ & $\mathrm{U}(1)_{B}$ \\
\hline$\Psi_{L}=\left(\begin{array}{c}\Psi_{L}^{0} \\
\Psi_{L}^{-}\end{array}\right)$ & 1 & 2 & $-\frac{1}{2}$ & $-\frac{3}{2}$ \\
$\Psi_{R}=\left(\begin{array}{c}\Psi_{R}^{0} \\
\Psi_{R}^{-}\end{array}\right)$ & 1 & 2 & $-\frac{1}{2}$ & $\frac{3}{2}$ \\
$\eta_{R}^{-}$ & 1 & 1 & -1 & $-\frac{3}{2}$ \\
$\eta_{L}^{-}$ & 1 & 1 & -1 & $\frac{3}{2}$ \\
$\chi_{R}^{0}$ & 1 & 1 & 0 & $-\frac{3}{2}$ \\
$\chi_{L}^{0}$ & 1 & 1 & 0 & $\frac{3}{2}$ \\
\hline \hline
\end{tabular}

fields, and it is a Majorana fermion. Recently, this model has been investigated in Ref. [45]. See also a recent study in Ref. [24] where one can have a similar model where the DM candidate is a Dirac fermion.

Model II: In the model proposed in Ref. [21], the anomalies are canceled with only four extra fields listed in Table II.

The Yukawa interactions needed to generate vectorlike masses for the new fermions are given by

$$
\begin{gathered}
\mathcal{L} \supset y_{1} \bar{\Psi}_{R} H \chi_{L}+y_{2} H^{\dagger} \Psi_{L} \chi_{L}+y_{3} H^{\dagger} \Sigma_{L} \Psi_{L}+y_{4} \bar{\Psi}_{R} \Sigma_{L} H \\
+\lambda_{1} \bar{\Psi}_{L} \Psi_{R} S_{B}+\frac{\lambda_{\chi}}{\sqrt{2}} \chi_{L} \chi_{L} S_{B}+\lambda_{\Sigma} \operatorname{Tr} \Sigma_{L}^{2} S_{B}+\text { H.c. }
\end{gathered}
$$

the DM is always a Majorana fermion, and it can be a linear combination of all neutral fields present in the theory. See Ref. [46] for a detailed phenomenological study of this model.

In these theories, the stability of the dark matter candidate is a natural consequence of the symmetry breaking. In this context, the local $\mathrm{U}(1)_{B}$ is broken to a $Z_{2}$ discrete symmetry which protects the stability of the DM candidate. It is important to mention that the stability of the

TABLE II. Fermionic representations in the model proposed in Ref. [21].

\begin{tabular}{lcccc}
\hline \hline Fields & $\mathrm{SU}(3)_{C}$ & $\mathrm{SU}(2)_{L}$ & $\mathrm{U}(1)_{Y}$ & $\mathrm{U}(1)_{B}$ \\
\hline$\Psi_{L}=\left(\begin{array}{l}\Psi_{L}^{+} \\
\Psi_{L}^{0}\end{array}\right)$ & 1 & 2 & $\frac{1}{2}$ & $\frac{3}{2}$ \\
$\Psi_{R}=\left(\begin{array}{l}\Psi_{R}^{+} \\
\Psi_{R}^{0}\end{array}\right)$ & 1 & 2 & $\frac{1}{2}$ & $-\frac{3}{2}$ \\
$\Sigma_{L}=\frac{1}{\sqrt{2}}\left(\begin{array}{ll}\Sigma_{L}^{0} & \sqrt{2} \Sigma_{L}^{+} \\
\sqrt{2} \Sigma_{L}^{-} & -\Sigma_{L}^{0}\end{array}\right)$ & 1 & 3 & 0 & $-\frac{3}{2}$ \\
$\chi_{L}^{0}$ & 1 & 1 & 0 & $-\frac{3}{2}$ \\
\hline \hline
\end{tabular}




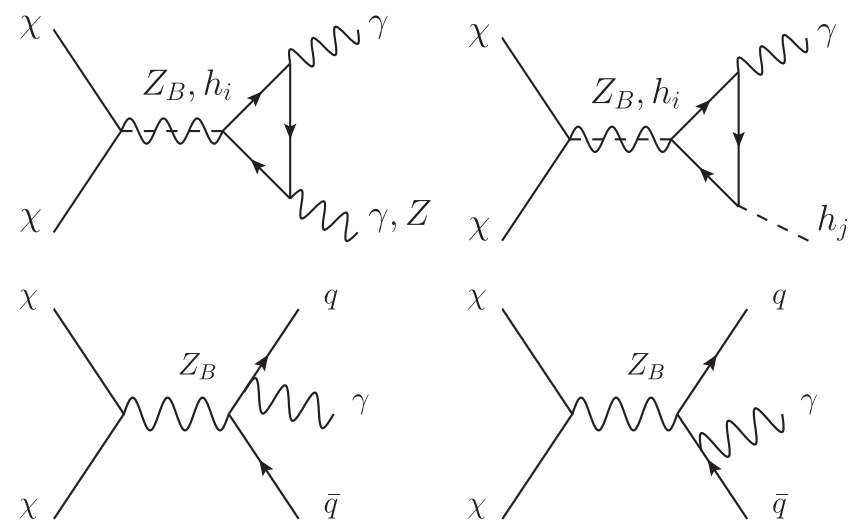

FIG. 5. Upper panel: Feynman graphs for the dark matter annihilation into gamma rays. Lower panel: Feynman graphs for the processes that contribute to final-state radiation.

DM candidate is never spoiled by any higher-dimensional operators one could write.

\section{B. Predictions for gamma lines}

The relevant interactions for the DM annihilation into gamma lines can be written as

$$
\begin{aligned}
\mathcal{L} \supset & -g_{B} \overline{f_{+}}\left(n_{V}^{f_{+}} \gamma^{\mu}+n_{A}^{f_{+}} \gamma^{\mu} \gamma^{5}\right) f_{+} Z_{\mu}^{B} \\
& -\frac{e}{\sin \theta_{W} \cos \theta_{W}} \overline{f_{+}}\left(g_{V}^{f} \gamma^{\mu}+g_{A}^{f} \gamma^{\mu} \gamma_{5}\right) f_{+} Z_{\mu},
\end{aligned}
$$

where $f_{+}$are the charged fermions entering in the loops shown in the upper panel of Fig. 5. This figure also shows the three different possibilities for the generation of gamma lines: $\chi \chi \rightarrow \gamma \gamma, \chi \chi \rightarrow Z \gamma$, and $\chi \chi \rightarrow h_{i} \gamma$.

\section{Gamma lines}

In these theories, the DM annihilation into a photon and a Higgs is velocity suppressed, but the cross sections for the gamma lines coming from $\chi \chi \rightarrow \gamma \gamma$ and $\chi \chi \rightarrow \gamma Z$ can be large. These cross sections are given by

$$
\begin{aligned}
\sigma v(\chi \chi \rightarrow \gamma \gamma)= & \frac{\alpha^{2}}{\pi^{3}} \frac{g_{B}^{4} n_{\chi}^{2} M_{\chi}^{2}}{M_{Z_{B}}^{4}} \frac{\left(4 M_{\chi}^{2}-M_{Z_{B}}^{2}\right)^{2}}{\left(4 M_{\chi}^{2}-M_{Z_{B}}^{2}\right)^{2}+\Gamma_{Z_{B}}^{2} M_{Z_{B}}^{2}} \\
& \times\left|\sum_{f_{+}} N_{c}^{f_{+}} n_{A}^{f_{+}} Q_{f_{+}}^{2} M_{f_{+}}^{2} C_{0}^{\gamma}\right|^{2}
\end{aligned}
$$

and

$$
\begin{aligned}
& \sigma v(\chi \chi \rightarrow \gamma Z) \\
& =\frac{\alpha^{2} g_{B}^{4} n_{\chi}^{2}}{32 \pi^{3} \sin ^{2} 2 \theta_{W}} \frac{\left(4 M_{\chi}^{2}-M_{Z}^{2}\right)^{3}}{\left(4 M_{\chi}^{2}-M_{Z_{B}}^{2}\right)^{2}+\Gamma_{Z_{B}}^{2} M_{Z_{B}}^{2}} \frac{\left(M_{Z_{B}}^{2}-4 M_{\chi}^{2}\right)^{2}}{M_{\chi}^{4} M_{Z_{B}}^{4}} \\
& \quad \times\left|\sum_{f} N_{c}^{f_{+}} Q_{f_{+}} n_{A}^{f_{+}} g_{V}^{f_{+}} 2 M_{f_{+}}^{2} C_{0}^{Z}\right|^{2},
\end{aligned}
$$

where $\alpha=e^{2} / 4 \pi, C_{0}^{A}=C_{0}\left(0, M_{A}^{2}, s ; M_{f}, M_{f}, M_{f}\right)$ is the Passarino-Veltman loop function as defined in Ref. [47] and $n_{\chi}$ is the dark matter charge under $\mathrm{U}(1)_{B}$. In order to predict the cross sections for these quantum mechanical processes, we need to use the interactions of the new fields required for anomaly cancellation.

Model I: In this case, as the following diagrams show,

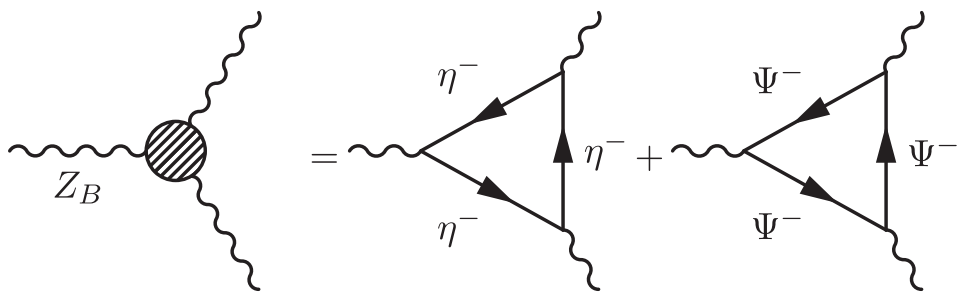

only the charged fields $\Psi^{-}$and $\eta^{-}$will contribute to the DM annihilation into gamma lines. The relevant Lagrangian in this case is given by

$$
\begin{aligned}
\mathcal{L}^{\mathrm{I}} \supset e \overline{\Psi^{-}} \not A \Psi^{-}+e \overline{\eta^{-}} A \eta^{-}+\frac{e}{\tan 2 \theta_{W}} \overline{\Psi^{-}} \not Z \Psi^{-} \\
\quad-e \tan \theta_{W} \overline{\eta^{-}} \not \eta^{-}-\frac{3}{2} g_{B} \overline{\Psi^{-}} Z_{B} \gamma_{5} \Psi^{-}+\frac{3}{2} g_{B} \overline{\eta^{-}} Z_{B} \gamma_{5} \eta^{-},
\end{aligned}
$$

from which one can read the couplings to the $Z_{B}$ gauge boson: $n_{V}^{\Psi^{-}}=0, n_{A}^{\Psi^{-}}=3 / 2, n_{V}^{\eta^{-}}=0$, and $n_{A}^{\eta^{-}}=-3 / 2$, and the couplings to the $Z$ boson: $g_{V}^{\Psi^{-}}=-\frac{1}{2}, g_{A}^{\Psi^{-}}=0$,
$g_{V}^{\eta^{-}}=\sin ^{2} \theta_{W}$, and $g_{A}^{\eta^{-}}=0$. In this scenario, in the limit where the couplings $y_{1}=y_{2}=y_{3}=y_{4}=0$, the masses for the new charged fermions read as

$$
M_{\Psi^{-}}=\frac{\lambda_{1} M_{Z_{B}}}{3 \sqrt{2} g_{B}}, \quad M_{\eta^{-}}=\frac{\lambda_{2} M_{Z_{B}}}{3 \sqrt{2} g_{B}} .
$$

The upper bound on $M_{Z_{B}}$ derived from relic density constraints defines a global upper bound for the new charged fermion masses, since the Yukawa couplings $\lambda_{1}$ and $\lambda_{2}$ are bounded by perturbativity. Moreover, the perturbative bounds define an upper bound for each mass of the new mediator. Hence, for a given $M_{Z_{B}}$ and $M_{\chi}$, the masses of the charged fermions are constrained to be in the following range: 


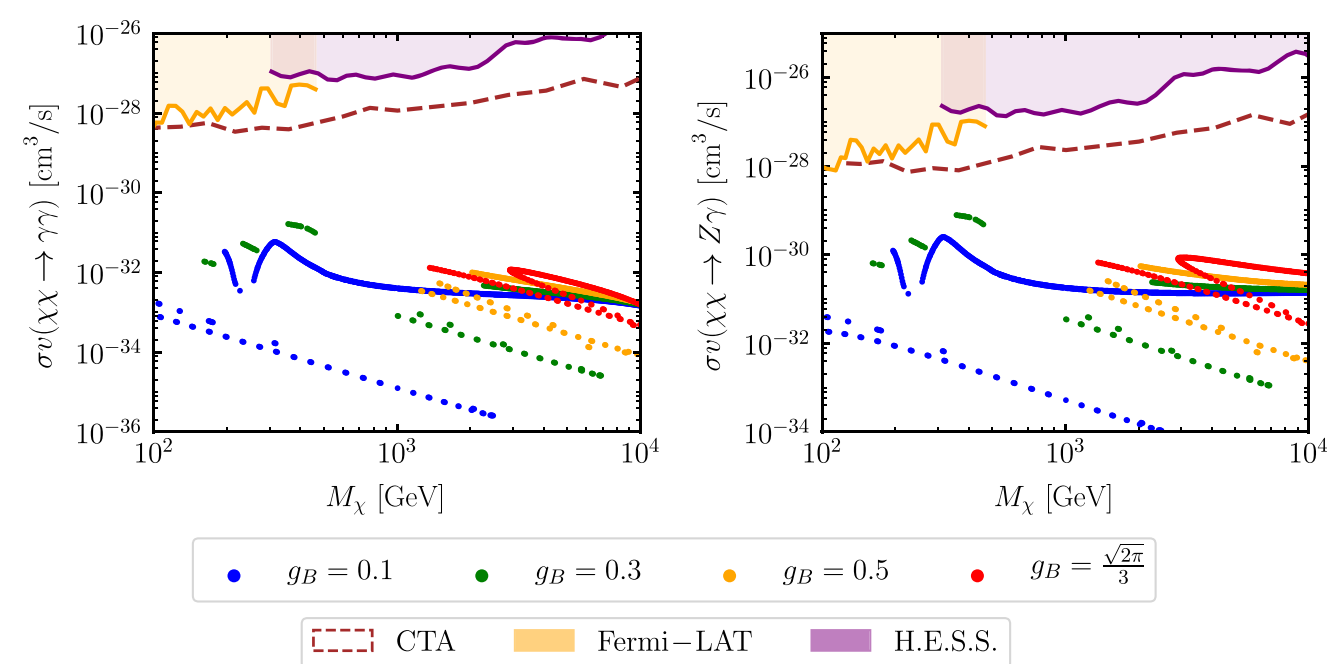

FIG. 6. Predictions for the DM annihilation into two photons (left panel) and into a photon and a $Z$ boson (right panel) in the context of Model I. We set $M_{h_{2}}=500 \mathrm{GeV}$ and $\theta_{B}=0$. The value of $M_{Z_{B}}$ is chosen such that every point satisfies $\Omega_{\mathrm{DM}} h^{2}=0.1197 \pm 0.0022$, and different colors correspond to different values of the gauge coupling $g_{B}$. All points shown pass LHC and direct detection constraints. The regions colored in yellow and purple show the excluded parameter space by Fermi-LAT [48,49] and H.E.S.S. [50], respectively. The dashed brown line shows the projected sensitivity from the CTA Collaboration [51].

$$
M_{\chi}<M_{f^{+}} \leq \frac{2 \sqrt{\pi}}{3 g_{B}} M_{Z_{B}}
$$

where $f^{+}$symbolizes any of the charged new fermions; the lower bound comes from ensuring the stability of the dark matter candidate, whereas the upper bound comes from perturbativity on the Yukawa couplings. We note that the dark matter candidate and the charged fermions satisfy the same perturbative bound.

In Fig. 6, we present our predictions for the DM annihilation into gamma lines for different choices of $g_{B}$ in the context of Model I for the two relevant annihilation channels. All the points shown saturate the relic abundance, $\Omega_{\mathrm{DM}} h^{2}=0.1197 \pm 0.0022$, and satisfy LHC and direct detection constraints. From Eqs. (24) and (25), we can see that when the charged fermions have the same mass, the cross section vanishes. We have taken the maximum mass splitting by choosing $M_{\Psi^{-}}=1.3 M_{\chi}$, in order to ensure the stability of the dark matter candidate, and $M_{\eta^{-}}=2 \sqrt{\pi} M_{Z_{B}} /$ $3 g_{B}$, i.e., the largest value allowed by perturbativity. Additionally, for each point we check that the perturbativity constraint, Eq. (28), is satisfied.

Model II: In this context, the relevant Lagrangian for the gamma lines reads as

$$
\begin{aligned}
\mathcal{L}^{\mathrm{II}} \supset & -e \overline{\Sigma^{+}} \not A \Sigma^{+}-e \overline{\Psi^{+}} \not \mathcal{A} \Psi^{+}-\frac{e}{\tan \theta_{W}} \overline{\Sigma^{+}} \not Z \Sigma^{+} \\
& -\frac{e}{\tan 2 \theta_{W}} \overline{\Psi^{+}} \not Z \Psi^{+}+\frac{3}{2} g_{B} \overline{\Psi^{+}} Z_{B} \gamma_{5} \Psi^{+} \\
& -\frac{3}{2} g_{B} \overline{\Sigma^{+}} Z_{B} \gamma_{5} \Sigma^{+},
\end{aligned}
$$

where $\Sigma^{+}$and $\Psi^{+}$correspond to the fields contributing to DM annihilation into gamma lines.
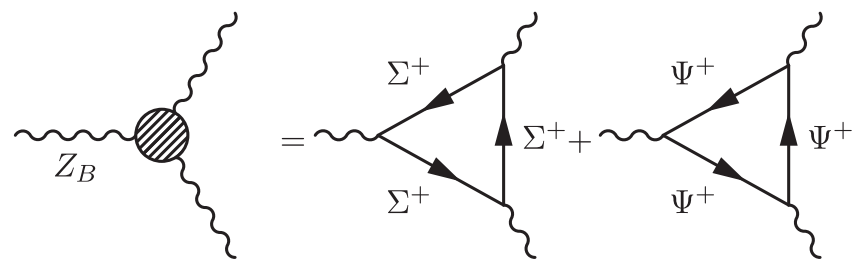

From the above Lagrangian, one can identify the couplings to the $Z_{B}$ boson: $n_{V}^{\Psi^{+}}=0, n_{A}^{\Psi^{+}}=-\frac{3}{2}, n_{V}^{\Sigma^{+}}=0$, $n_{A}^{\Sigma^{+}}=\frac{3}{2}$, and the couplings to the $Z$ boson: $g_{V}^{\Psi^{+}}=\frac{1}{2}$, $g_{A}^{\Psi^{+}}=0, g_{V}^{\Sigma^{+}}=\cos ^{2} \theta_{W}$, and $g_{A}^{\Sigma^{+}}=0$. In this scenario, in the limit where the couplings $y_{1}=y_{2}=y_{3}=y_{4}=0$, the masses for the new charged fermions read as

$$
M_{\Psi^{+}}=\frac{\lambda_{1} M_{Z_{B}}}{3 \sqrt{2} g_{B}}, \quad M_{\Sigma^{+}}=\frac{\sqrt{2} \lambda_{\Sigma} M_{Z_{B}}}{3 g_{B}} .
$$

As in the previous case, here there is also a global upper bound on the charged fermion masses defined by the upper bound on the $M_{Z_{B}}$ and the perturbative bounds of the Yukawa couplings. As already discussed, the masses of the charged fermions must be in the range defined by Eq. (28) to ensure the stability of the dark matter candidate and the perturbativity of the Yukawa couplings $\lambda_{\Sigma}$ and $\lambda_{1}$.

In Fig. 7, we present our predictions for the DM annihilation into gamma lines for different choices of $g_{B}$ in the context of Model II. As in Model I, the points satisfy $\Omega_{\mathrm{DM}} h^{2}=0.1197 \pm 0.0022$, and the masses of the charged 


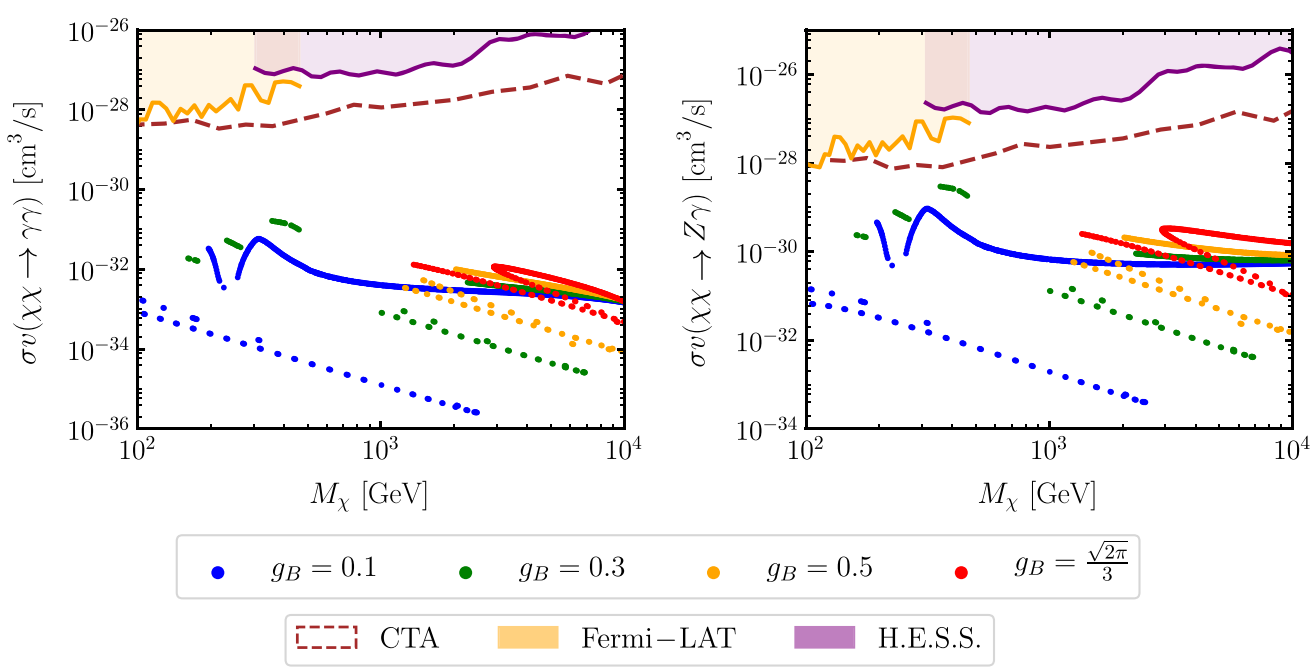

FIG. 7. Predictions for the DM annihilation into two photons (left panel) and into a photon and a $Z$ boson (right panel) in the context of Model II. We set $M_{h_{2}}=500 \mathrm{GeV}$ and $\theta_{B}=0$. The value of $M_{Z_{B}}$ is chosen such that every point satisfies $\Omega_{\mathrm{DM}} h^{2}=0.1197 \pm 0.0022$, and different colors correspond to different values of the gauge coupling $g_{B}$. All points shown pass LHC and direct detection constraints. The regions colored in yellow and purple show the excluded parameter space by Fermi-LAT [48,49] and H.E.S.S. [50], respectively. The dashed brown line shows the projected sensitivity from the CTA Collaboration [51].

fermions are chosen to maximize the splitting among them: $M_{\Psi^{+}}=1.3 M_{\chi}$ and $M_{\Sigma^{+}}=2 \sqrt{\pi} M_{Z_{B}} / 3 g_{B}$.

Regarding gamma lines, the only difference between the two models is the coupling of one of the extra fields to the $Z$ boson. Consequently, for the same choice of masses, the $\gamma \gamma$ line is the same in the context of both models, but there are slight differences for the $\gamma Z$ line. The right panels in Figs. 6 and 7 show the cross sections for the $Z \gamma$ line, and as can be appreciated, for Model I the predictions are slightly weaker than for Model II (due to the fact that $\eta^{-} \eta^{-} Z$ coupling is suppressed by $\tan ^{2} \theta_{W}$ with respect to the $\Sigma^{+} \Sigma^{+} Z$ coupling, as shown in the above Lagrangians). The left panels are the same because the $\gamma \gamma$ line dominates over the $Z \gamma$ line, so the predictions are the same for both models. The signals displayed in Figs. 6 and 7 are, unfortunately, in a region that remains a few orders of magnitude below the current experimental sensitivity. However, we would like to remark that these two $\mathrm{U}(1)_{B}$ models are two of the most motivated SM extensions that predict axial interactions between charged fermions with the new mediator that allow predictions for the DM annihilation into gamma lines, although being small, to be different from zero. Furthermore, there is hope that future telescopes can test or rule out these predictions.

\section{Final-state radiation processes}

As pointed out in Ref. [19], a Majorana candidate for dark matter is crucial in order to see its annihilation into gamma lines. It is well known that the generation of gamma lines takes place through a quantum mechanical process, whereas the processes contributing to the continuum spectrum are, in general, tree-level processes (see lower panel of Fig. 5). However, if the DM candidate is a Majorana fermion, the amplitude for the processes contributing to the final-state radiation can be expanded as

$$
|\mathcal{M}|_{\mathrm{FSR}}^{2}=\frac{M_{q}^{2}}{M_{Z_{B}}^{2}} A+v^{2} B+\mathcal{O}\left(v^{4}\right),
$$

where $A$ and $B$ are coefficients defined at the end of this section. From here, one can see that they are either velocity or mass $\left(M_{q} / M_{Z_{B}}\right)$ suppressed. Therefore, in this case, one can hope to distinguish the gamma lines from the continuum spectrum. The coefficients that parametrize the expansion in velocity for the FSR processes are given by

$$
\begin{aligned}
A= & 12 \pi \alpha g_{B}^{4} Q_{q}^{2}\left(M_{Z_{B}}^{2}-4 M_{\chi}^{2}\right)^{2} \frac{\left(E_{q}+E_{\gamma}-M_{\chi}\right)^{2}\left(2\left(E_{q}-M_{\chi}\right)\left(E_{q}+E_{\gamma}-M_{\chi}\right)-3 M_{q}^{2}\right)}{M_{Z_{B}}^{2}\left(E_{q}-M_{\chi}\right)^{2}\left(E_{q}+E_{\gamma}-M_{\chi}\right)^{2}\left(\left(4 M_{\chi}^{2}-M_{Z_{B}}^{2}\right)^{2}+\Gamma_{Z_{B}}^{2} M_{Z_{B}}^{2}\right)}, \\
B= & 12 \pi \alpha g_{B}^{4} M_{\chi}^{2} Q_{q}^{2} \\
& \times \frac{\left(2 E_{q} M_{\chi}\left(E_{\gamma}^{2}-3 E_{\gamma} M_{\chi}+2 M_{\chi}^{2}\right)-2 E_{q}^{4}-2 E_{q}^{3}\left(E_{\gamma}-2 M_{\chi}\right)-E_{q}^{2}\left(E_{\gamma}^{2}-6 E_{\gamma} M_{\chi}+6 M_{\chi}^{2}\right)-2 M_{\chi}^{2}\left(E_{\gamma}-M_{\chi}\right)^{2}\right)}{M_{Z_{B}}^{2}\left(E_{q}+E_{\gamma}-M_{\chi}\right)^{2}\left(\left(4 M_{\chi}^{2}-M_{Z_{B}}^{2}\right)^{2}+\Gamma_{Z_{B}}^{2} M_{Z_{B}}^{2}\right)} .
\end{aligned}
$$




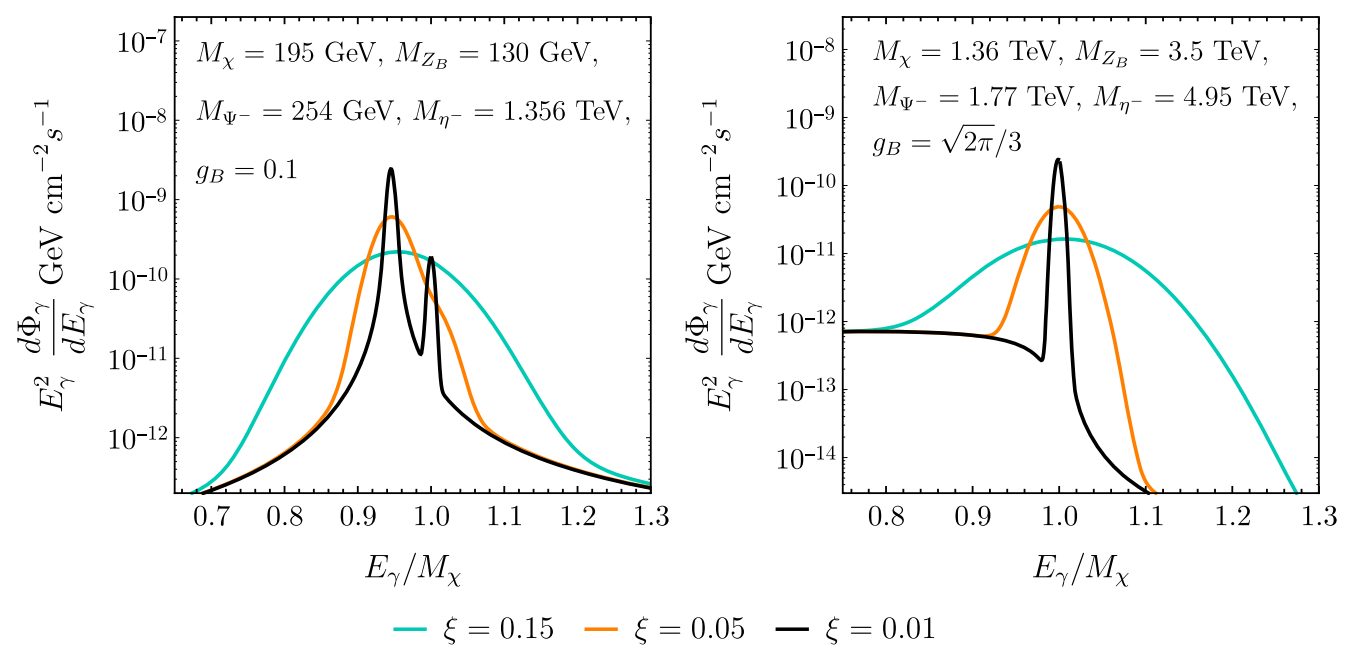

FIG. 8. The differential spectrum of the dark matter annihilation into gamma rays for two different scenarios that satisfy the relic abundance measured by the Planck satellite $\Omega_{\mathrm{DM}} h^{2}=0.1197 \pm 0.0022$ [27] in Model I. We set $M_{h_{2}}=500 \mathrm{GeV}$ and $\theta_{B}=0$. Lines with different colors correspond to different energy resolutions $\xi=\{0.01,0.05,0.15\}$.

\section{Gamma line spectra}

The prediction for the gamma-ray flux is given by

$$
\frac{d \Phi_{\gamma}}{d E_{\gamma}}=\frac{n_{\gamma}}{8 \pi M_{\chi}^{2}} \frac{d\left\langle\sigma v_{\text {rel }}\right\rangle}{d E_{\gamma}} J_{\mathrm{ann}}=\frac{n_{\gamma}\left\langle\sigma v_{\mathrm{rel}}\right\rangle}{8 \pi M_{\chi}^{2}} \frac{d N}{d E_{\gamma}} J_{\mathrm{ann}}
$$

where $n_{\gamma}$ is a multiplicity factor, $n_{\gamma}=2(=1)$, for the $\gamma \gamma(\gamma Z)$ annihilation channel. In order to compute the total flux, we include the gamma lines cross section and the final-state radiation. The $J$ factor, $J_{\text {ann }}$, encodes all astrophysical assumptions made regarding the dark matter
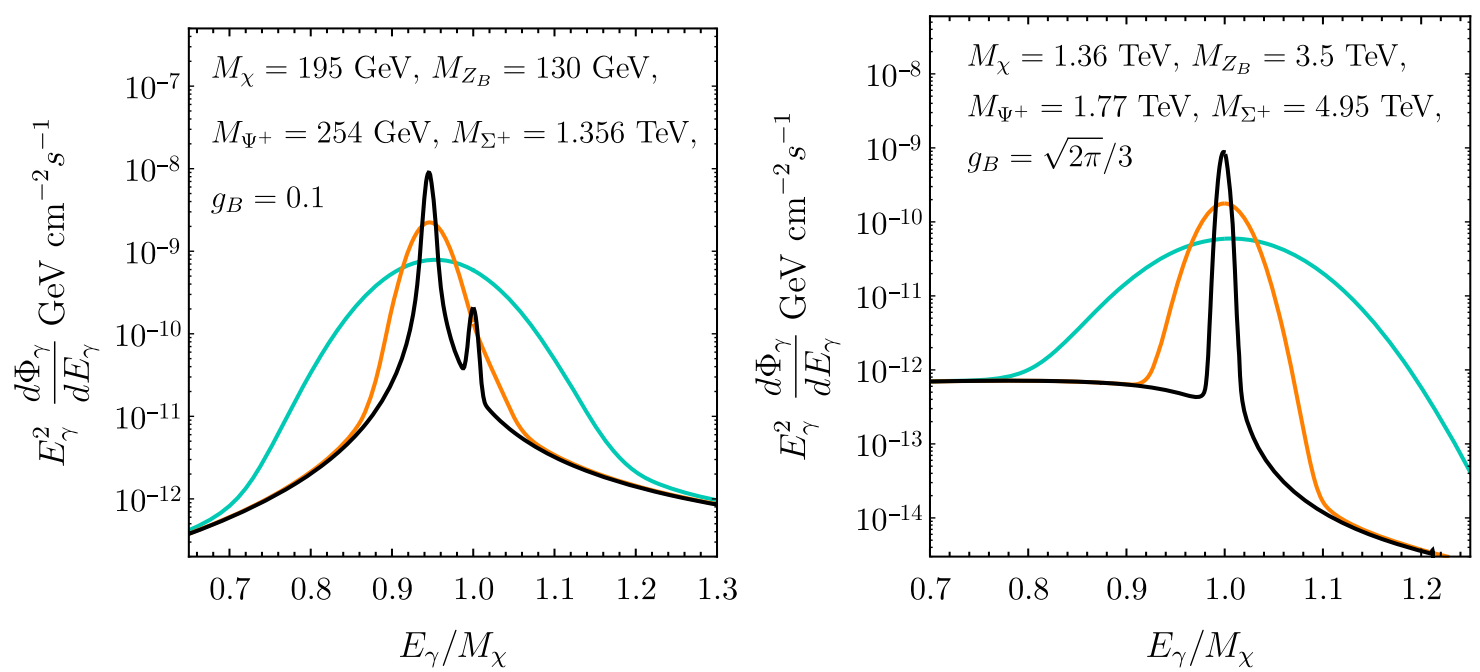

$-\xi=0.15-\xi=0.05-\xi=0.01$

FIG. 9. The differential spectrum of the dark matter annihilation into gamma rays for two different scenarios that satisfy the relic abundance measured by the Planck satellite $\Omega_{\mathrm{DM}} h^{2}=0.1197 \pm 0.0022$ [27] in Model II. We set $M_{h_{2}}=500 \mathrm{GeV}$ and $\theta_{B}=0$. Lines with different colors correspond to different energy resolutions $\xi=\{0.01,0.05,0.15\}$. 
for the $Z \gamma$ line. In order to account for the energy resolution of the detector, we apply a Gaussian function,

$$
G\left(E_{\gamma}, \xi / \omega, E_{0}\right)=\frac{1}{\sqrt{2 \pi} E_{0}(\xi / \omega)} e^{-\frac{\left(E_{\gamma}-E_{0}\right)^{2}}{2 E_{0}^{2}(\xi / \omega)^{2}}},
$$

where $\xi$ is the energy resolution and $\omega=2 \sqrt{2 \log 2} \approx 2.35$ determines the full width at half maximum, with the standard deviation given by $\sigma_{0}=E_{0} \xi / w$.

In Fig. 8, we present our results for the differential spectrum of dark matter annihilation into gamma rays for parameters satisfying the relic abundance, $\Omega_{\mathrm{DM}} h^{2}=$ $0.1197 \pm 0.0022$, in Model I and different values of the energy resolution $\xi=\{0.01,0.05,0.15\}$. As can be appreciated, the gamma lines can be easily distinguished from the continuum spectrum because the FSR processes are highly suppressed. However, for gamma-ray telescopes with energy resolution larger than $5 \%$, it is impossible to distinguish the gamma lines between the $\chi \chi \rightarrow \gamma \gamma$ and $\chi \chi \rightarrow \gamma Z$ annihilation channels. In Fig. 9, we show the predictions for the differential spectrum of the dark matter annihilation into gamma rays for different values saturating the relic abundance in two different scenarios in Model II. The predictions for the gamma lines in Models I and II are very striking but very similar, and hence, collider searches are needed in order to distinguish between the two models. This is the goal of our future publication.

\section{SUMMARY}

In this work, we investigated the properties of a Majorana dark matter candidate predicted in anomaly-free theories. In these theories, the dark matter mass is defined by the new symmetry breaking scale, and its stability is ensured by a remnant discrete symmetry after the gauge symmetry is broken. We focus our study in the context of simple theories where the baryon number is a local gauge symmetry spontaneously broken at the low scale. Our dark matter candidate is a Majorana fermion charged under the $\mathrm{U}(1)_{B}$ gauge group, and hence, it has an axial coupling to the new gauge boson $Z_{B}$. We performed the calculation of the relic density and discussed experimental constraints coming from the LHC and direct detection experiments. The axial coupling implies that for vanishing Higgs mixing the dark matter-nucleon cross section is velocity suppressed. Therefore, the bounds from direct detection experiments only become relevant for large Higgs mixing. However, dijet resonance searches at the LHC will further probe the regions in which $M_{Z_{B}}$ lies around the electroweak scale. Moreover, we found that the cosmological constraint on the dark matter relic density, $\Omega_{\mathrm{DM}} h^{2} \leq 0.12$, implies that the new gauge boson and the dark matter masses must be below the multi-TeV scale, i.e., $M_{Z_{B}} \lesssim 28 \mathrm{TeV}$ and $M_{\chi} \lesssim 34 \mathrm{TeV}$.

The new fermions needed for anomaly cancellation in these theories are chiral under $\mathrm{U}(1)_{B}$ and acquire their masses after this symmetry has been spontaneously broken. Therefore, the upper bound on the baryon number violation scale translates as an upper bound on their masses. These upper bounds tell us that there is hope to detect this new sector of the theory at the LHC or at future particle colliders. Regarding indirect detection, the dark matter axial coupling implies that the final-state radiation is velocity suppressed. Consequently, the gamma lines from dark matter annihilation can be distinguished from the continuum. We would like to emphasize that consistent completions of simplified models of dark matter give rise to interesting phenomenology; namely, gamma line features that can be probed at future gamma-ray telescopes. Due to the gauge anomaly conditions, these signatures cannot be computed within a simplified model where the anomalies are not canceled. Our predictions for the gamma lines could be crucial to testing these dark matter theories in the future.

\section{ACKNOWLEDGMENTS}

The work of C. M. has been supported in part by Grants No. FPA2014-53631-C2-1-P, No. FPA2017-84445-P, and No. SEV-2014- 0398 (AEI/ERDF, EU), and by a La CaixaSevero Ochoa scholarship.
[1] M. Beltran, D. Hooper, E. W. Kolb, and Z. C. Krusberg, Deducing the nature of dark matter from direct and indirect detection experiments in the absence of collider signatures of new physics, Phys. Rev. D 80, 043509 (2009).

[2] Q.-H. Cao, C.-R. Chen, C.S. Li, and H. Zhang, Effective dark matter model: Relic density, CDMS II, Fermi LAT and LHC, J. High Energy Phys. 08 (2011) 018.

[3] M. Beltran, D. Hooper, E. W. Kolb, Z. A. C. Krusberg, and T. M. P. Tait, Maverick dark matter at colliders, J. High Energy Phys. 09 (2010) 037.
[4] J. Goodman, M. Ibe, A. Rajaraman, W. Shepherd, T. M. P. Tait, and H.-Bo. Yu, Constraints on light Majorana dark matter from colliders, Phys. Lett. B 695, 185 (2011).

[5] Y. Bai, P. J. Fox, and R. Harnik, The Tevatron at the frontier of dark matter direct detection, J. High Energy Phys. 12 (2010) 048.

[6] G. Busoni, A. De Simone, E. Morgante, and A. Riotto, On the validity of the effective field theory for dark matter searches at the LHC, Phys. Lett. B 728, 412 (2014). 
[7] O. Buchmueller, M. J. Dolan, and C. McCabe, Beyond effective field theory for dark matter searches at the LHC, J. High Energy Phys. 01 (2014) 025.

[8] G. Busoni, A. De Simone, J. Gramling, E. Morgante, and A. Riotto, On the validity of the effective field theory for dark matter searches at the LHC, Part II: Complete analysis for the s-channel, J. Cosmol. Astropart. Phys. 06 (2014) 060.

[9] O. Buchmueller, M. J. Dolan, S. A. Malik, and C. McCabe, Characterising dark matter searches at colliders and direct detection experiments: Vector mediators, J. High Energy Phys. 01 (2015) 037.

[10] M. R. Buckley, D. Feld, and D. Goncalves, Scalar simplified models for dark matter, Phys. Rev. D 91, 015017 (2015).

[11] P. Harris, V. V. Khoze, M. Spannowsky, and C. Williams, Constraining dark sectors at colliders: Beyond the effective theory approach, Phys. Rev. D 91, 055009 (2015).

[12] U. Haisch and E. Re, Simplified dark matter top-quark interactions at the LHC, J. High Energy Phys. 06 (2015) 078.

[13] M. Duerr, P. Fileviez Perez, and M. B. Wise, Gauge Theory for Baryon and Lepton Numbers with Leptoquarks, Phys. Rev. Lett. 110, 231801 (2013).

[14] F. Kahlhoefer, K. Schmidt-Hoberg, T. Schwetz, and S. Vogl, Implications of unitarity and gauge invariance for simplified dark matter models, J. High Energy Phys. 02 (2016) 016.

[15] A. Ismail, W.-Y. Keung, K.-H. Tsao, and J. Unwin, Axial vector $Z^{\prime}$ and anomaly cancellation, Nucl. Phys. B918, 220 (2017).

[16] J. Ellis, M. Fairbairn, and P. Tunney, Anomaly-free dark matter models are not so simple, J. High Energy Phys. 08 (2017) 053.

[17] Y. Cui and F. D'Eramo, Surprises from complete vector portal theories: New insights into the dark sector and its interplay with Higgs physics, Phys. Rev. D 96, 095006 (2017).

[18] M. Duerr, P. Fileviez Perez, and J. Smirnov, Simplified Dirac dark matter models and gamma-ray lines, Phys. Rev. D 92, 083521 (2015).

[19] P. F. Pérez and C. Murgui, Gamma lines from the hidden sector, arXiv:1901.10483.

[20] P. F. Pérez and C. Murgui, Dark matter and the seesaw scale, Phys. Rev. D 98, 055008 (2018).

[21] P. Fileviez Perez, S. Ohmer, and H. H. Patel, Minimal theory for lepto-baryons, Phys. Lett. B 735, 283 (2014).

[22] P. Fileviez Perez, New paradigm for baryon and lepton number violation, Phys. Rep. 597, 1 (2015).

[23] P. Fileviez Perez and M. B. Wise, Breaking local baryon and lepton number at the $\mathrm{TeV}$ scale, J. High Energy Phys. 08 (2011) 068.

[24] P. F. Pérez, E. Golias, R.-H. Li, and C. Murgui, Leptophobic dark matter and the baryon number violation scale, Phys. Rev. D 99, 035009 (2019).

[25] A. Semenov, LanHEP: A package for automatic generation of Feynman rules from the Lagrangian, Version 3.2, Comput. Phys. Commun. 201, 167 (2016).

[26] G. Bélanger, F. Boudjema, A. Goudelis, A. Pukhov, and B. Zaldivar, MICROMEGAs5.0: Freeze-in, Comput. Phys. Commun. 231, 173 (2018).
[27] P. A. R. Ade et al. (Planck Collaboration), Planck 2015 results: XIII. Cosmological parameters, Astron. Astrophys. 594, A13 (2016).

[28] J. Ellis, M. Fairbairn, and P. Tunney, Phenomenological constraints on anomaly-free dark matter models, arXiv: 1807.02503 .

[29] M. Hoferichter, P. Klos, J. Menéndez, and A. Schwenk, Improved Limits for Higgs-Portal Dark Matter from LHC Searches, Phys. Rev. Lett. 119, 181803 (2017).

[30] M. Duerr, P. F. Pérez, and J. Smirnov, Baryonic Higgs at the LHC, J. High Energy Phys. 09 (2017) 093.

[31] A. Ilnicka, T. Robens, and T. Stefaniak, Constraining extended scalar sectors at the LHC and beyond, Mod. Phys. Lett. A 33, 1830007 (2018).

[32] E. Aprile et al. (XENON Collaboration), First Dark Matter Search Results from the XENON1T Experiment, Phys. Rev. Lett. 119, 181301 (2017).

[33] E. Aprile et al. (XENON Collaboration), Dark Matter Search Results from a One Ton-Year Exposure of XENON1T, Phys. Rev. Lett. 121, 111302 (2018).

[34] E. Aprile et al. (XENON Collaboration), Physics reach of the XENON1T dark matter experiment, J. Cosmol. Astropart. Phys. 04 (2016) 027.

[35] J. Billard, L. Strigari, and E. Figueroa-Feliciano, Implication of neutrino backgrounds on the reach of next generation dark matter direct detection experiments, Phys. Rev. D 89, 023524 (2014).

[36] T. Bringmann and C. Weniger, Gamma ray signals from dark matter: Concepts, status and prospects, Phys. Dark Universe 1, 194 (2012).

[37] M. Duerr, P. Fileviez Perez, and J. Smirnov, Gamma lines from Majorana dark matter, Phys. Rev. D 93, 023509 (2016).

[38] C. B. Jackson, G. Servant, G. Shaughnessy, T. M. P. Tait, and M. Taoso, Higgs in space!, J. Cosmol. Astropart. Phys. 04 (2010) 004.

[39] E. Dudas, Y. Mambrini, S. Pokorski, and A. Romagnoni, Extra U(1) as natural source of a monochromatic gamma ray line, J. High Energy Phys. 10 (2012) 123.

[40] C. B. Jackson, G. Servant, G. Shaughnessy, T. M. P. Tait, and M. Taoso, Gamma-ray lines and one-loop continuum from $s$-channel dark matter annihilations, J. Cosmol. Astropart. Phys. 07 (2013) 021.

[41] C. B. Jackson, G. Servant, G. Shaughnessy, T. M. P. Tait, and M. Taoso, Gamma rays from top-mediated dark matter annihilations, J. Cosmol. Astropart. Phys. 07 (2013) 006.

[42] E. Dudas, L. Heurtier, Y. Mambrini, and B. Zaldivar, Extra U(1), effective operators, anomalies and dark matter, J. High Energy Phys. 11 (2013) 083.

[43] D. Hooper, $Z^{\prime}$ mediated dark matter models for the galactic center gamma-ray excess, Phys. Rev. D 91, 035025 (2015).

[44] J. Kumar and D. Marfatia, Matrix element analyses of dark matter scattering and annihilation, Phys. Rev. D 88, 014035 (2013).

[45] S. Caron, J. A. Casas, J. Quilis, and R. Ruiz de Austri, Anomaly-free dark matter with harmless direct detection constraints, J. High Energy Phys. 12 (2018) 126.

[46] S. Ohmer and H. H. Patel, Leptobaryons as Majorana dark matter, Phys. Rev. D 92, 055020 (2015). 
[47] H. H. Patel, Package-X: A Mathematica package for the analytic calculation of one-loop integrals, Comput. Phys. Commun. 197, 276 (2015).

[48] M. Ackermann et al. (Fermi-LAT Collaboration), Updated search for spectral lines from Galactic dark matter interactions with pass 8 data from the Fermi large area telescope, Phys. Rev. D 91, 122002 (2015).

[49] M. Ackermann et al. (Fermi-LAT Collaboration), Search for gamma-ray spectral lines with the Fermi large area telescope and dark matter implications, Phys. Rev. D 88, 082002 (2013).

[50] A. Abramowski et al. (H.E.S.S. Collaboration), Search for Photon-Linelike Signatures from Dark Matter Annihilations with H.E.S.S., Phys. Rev. Lett. 110, 041301 (2013).

[51] B. S. Acharya et al. (CTA Consortium), Science with the Cherenkov Telescope Array (World Scientific, Singapore, 2018). 